Voix et Images

\title{
Guy Lafond et les Cloches d'autres mondes
}

\section{Thérèse Renaud}

Volume 4, numéro 2, décembre 1978

Guy Lafond

URI : https://id.erudit.org/iderudit/200152ar

DOI : https://doi.org/10.7202/200152ar

Aller au sommaire du numéro

Éditeur(s)

Les Presses de l'Université du Québec

ISSN

0318-9201 (imprimé)

1705-933X (numérique)

Découvrir la revue

Citer cet article

Renaud, T. (1978). Guy Lafond et les Cloches d'autres mondes. Voix et Images, 4(2), 205-216. https://doi.org/10.7202/200152ar d'utilisation que vous pouvez consulter en ligne.

https://apropos.erudit.org/fr/usagers/politique-dutilisation/ 


\section{Guy Lafond et \\ les Cloches d'autres mondes}

N'entre pas qui veut dans l'univers poétique de Guy Lafond, car il faut, pour aborder le poète, s'être abandonné avant toute chose au chant profond du poème, à l'agencement musical des mots qui deviendront le rythme de la phrase, qui à son tour suggérera l'image elle-même mise en position d'illustrer le sens. Et lorsque vous aurez l'impression d'être en possession du sens, une nouvelle énigme apparaîtra qui fera basculer l'ensemble dans un autre monde: celui de l'identification avec le cosmos; en fait, l'univers de notre conscience intuitive et créatrice: «Involucré dans la hanche du ciel, à la rigueur aucune, l'œil est un trou béant». On pense aux trous noirs découverts par les astro-physiciens.

Je recommande à tout nouveau lecteur de ne pas chercher à comprendre le poème de prime abord, mais de s'abandonner à la sourde mélodie du chant. Alors qu'il ne discernera pas encore à quelle réalité s'adresse le poète, il se passera quelque chose d'étrange et la beauté du poème jaillira dans sa transparence lumineuse. Petit à petit le chant transformera notre incrédibilité en une forme nouvelle et l'on sera à son tour aspiré par le poème.

\section{Les cloches d'autres mondes}

Le recueil, ou plutôt le poème, se divise en trois parties avec prologue. J'essayerai de dégager les étapes de cette épopée lyrique, et pour ce faire, j'ai choisi de commenter à la manière d'un dialogue continu l'itinéraire intérieur du poète.

Le prologue, "Entre lire et Orphée", se déroule en quatre temps; le poète organise sa propre mise à mort dans un déchirant dilemme :

Le choix est inévitable. Tu es l'impossibilité du choix.

L'impossibilité est dans le choix, la cire du choix soumis à l'évidence.

A la croix ${ }^{1}(13)$ 
Mais dans ce choix impossible et cependant inévitable, "un risque, la vie est péril de vie. II faut mourir à l'unanime et naître à l'inverse." (p. 16). Demeure le nœud, le cœur du choix, tantôt convoité ou mis en échec:

Le passé est à venir, le nœud est sans cesse.

Soleil d'outre-azur. Le ciel partout à la rencontre d'un ciel. (17)

Plus tard, lorsque le poète aura accepté la dérive de par le fait même de son identité - «les racines entravent» - il trouvera une sorte de sérénité dans l'acceptation:

Et vivre.

Un lieu de nulle demande. D'exigence je me lie. (18)

Dans un ultime espoir il franchira cette première étape :

La nuit déparée, celle-là est douce. Ah, connaître, mon repos!

D'ombre.

De sceurs.

Fleurs d'ombre. (18)

La première partie du recueil, intitulée "l'Aube livrée", aborde l'essence du détachement: “un essaim d'adieux où rêve une présence» (p. 21).

Le phrasé possède une musicalité ondoyante, parfois abrupte; on sent le ressac de la brise fine. Alors que tout invite à rester, il faut renoncer:

L'arrogance du regret

a couché le soleil

Depuis je bois

hors des musiques

la vertu plus douce que l'amorce d'un songe. (22)

Car l'appel est le plus fort, et lors même que l'on ignore ou que l'on voudrait ignorer ce qui semble inéluctable:

La mort allège l'amour

la nuit trop ancienne des aurores

Le rêve inscrit

l'appel fuyant des rives

Et le poème exalte

l'heure désappropriée. (27)

Alors que rien ne l'indiffère, que tout l'invite ou le retient, la nuit obscure fait son apparition :

«Je te délie

dans le recueillement de l'oubli

où je vis de mort muette". 
Fontaines, fontaines

Opales dévorantes

Gerbes! (28)

Quel avenir est réservé au poète résigné à poursuivre le chemin? "L'horizon est un sourire distrait à nulle raison" (p. 29). II vit son écartèlement comme une seconde mort, car il sait que la délivrance est à ce prix; "la mort erre parmi les nénuphars", «mais je sais la nuit pendue à la nuit" (p. 31).

Furtivement, il aborde l'autre rive :

L'oiseau boit le poids de son vol

la nuit le jour ébloui

Dans l'œil s'effrite le miroir

et la parole s'abreuve de saisons. (32)

Et tel enfant qui sortant du ventre de sa mère, aura à assumer un nouvel environnement, le poète assiste à cette nouvelle naissance, à cette conscience renouvelée :

Un sourire frais s'élève

dans le regard fugace

de sources imprévues

médusé. (36)

Il demeure un instant pétrifié par ce qu'il entrevoit comme possible:

O nuit sereine des aurores! (37)

Alors le passé s'abolit :

Je pendrai la parole ancienne

comme des pans de mer sur la soie vive. (38)

II termine alors qu'il sent le détachement accompli :

Aux confins

quatre archanges d'or

veillent. (39)

Le deuxième chant (qui donne son titre au recueil) semblera à première vue d'une lecture plus aisée. Le phrasé est mélodieux, parfois d'une musicalité proche du suave :

Faste de la pierre qui ne termine jamais

Ronde et lisse comme le bonheur

Présences! Vents! (43)

Comme ce jugement semblera dérisoire après relectures! Car plus on avance dans l'approche du poète, plus le décryptage de l'œuvre est exigeant. Guy Lafond sait très bien de quoi il parle et il livre sa connaissance des symboles à travers une profondeur de vue et de vécu sur l'être, ce qui confère à l'œuvre une grande densité. J'accorderai une attention particulière à ce deuxième chant à cause de la difficulté d'approche dans la simplicité même du phrasé. 
Le poète est ici enlisé dans le néant de deux mondes. L'un, qu'il a quitté, mais dont le souvenir s'inscrit au vif de la chair:

... et l'enfant s'avance dans la mer,

noyé par l'écho des miroirs où sommeillent

les cloches d'autres mondes. (44)

Il s'abandonne tout à fait à cet autre monde :

«Je n'emporte pas d'adieu, dit l'enfant, ni même une mémoire. J'allonge un bras douloureux dans l'absence de parfums pour traverser les silences lourds de fidelité, et marcher en une terre immense de fleurs " (45)

II faut bien comprendre la profondeur cachée de ce volet. Le poète ne s'accorde aucune tricherie, aucun adieu dans aucune mémoire; pour ce faire, il «allonge un bras douloureux", n'escomptant aucune joie («dans l'absence de parfums"), et cependant il demeurera fidèle à l'appel malgré les silences et les beautés secrètes ( "marcher en une terre immense de fleurs ") qui ne le touchent pas encore.

II décrit l'aridité de sa situation dans le volet suivant:

Belles dents de la mort

Violence inespérée du néant

Et sous la paupière du jour

L'écume éclatante de la nuit reconnue

et il conclut :

La nuit aspire les aurores

elle apaise les seuils. (45)

Cependant il faut secouer tout cela et renoncer à l'espoir pour luimême dans une égalité d'humeur :

La perle noire conjure les saisons.

Lorsqu'aux confins du sommeil la pierre lumineuse

s'enfonce dans la nuit, aussi sûre que la racine

dans un sol propice, elle puise au sourire une eau

accomplie, elle fleurit en froide sérénité. (46)

Et le poète recréé reprend:

"Tel est le jour, dit l'enfant, le champ incalculable des moissons; telle est l'offrande du jour, et la soumission de l'heure. " (46)

Un nouvel espoir est donné :

«Je t'accompagne plus loin que les magies, en un lieu où la terre s'abîme de terre, où le ciel s'épuise de ciel, où la main repose les vents. " (47)

Maintenant le départ est sans espoir de retour cependant qu'il reconnaît toutes les attaches qui le relient au passé; il ne craint plus les velléités de l'âme ("les hommes cueillent des larmes avares"):

"Je reviens, et ne serai jamais revenu.

J'habite un voile clos de matins. " (48) 
Soudain, dans ce désert, la conscience est illuminée :

Minuit! la foudre myriade éblouit l'astre instantané; la terre danse au feu intime. L'imprévu est le sceau de l'impression sur des toits de neige.

"Silence, dit l'enfant, tu foules un hasard fragile. L'heure est un baiser; et la terre est immobile dans l'immobilité. " (51)

A partir de ce moment, aucun soubresaut du rêve ne viendra déranger l'aspiration du poète:

«Nais, à chaque embrasement, la même naissance.

Car la vie chevauche d'interminables embarquements, et minuit s'ancre à l'immuable éclosion. 》 (51)

Et cette réalité s'inscrit dans le cœur du poète au même instant que le don :

(Poète, la musique gît comme un livre délaissé dans le creux du souffle. Ne retiens ni l'œil, ni les lentes promesses. Enferme-les dans le don. (52)

L'être est dans l'égalité de lui-même et des actes et des choses :

"ll y aura de grandes diversions, des pluies d'un silence à l'autre, de grands chemins d'un midi à l'autre, la plaie des pas incessants. Et dans l'aróme du sourire, la douceur comme un rappel de cendres." (53)

Le poète se sentant habité par la création entière,

Mystérieuse voie où le mystère s'évanouit.

La dernière image est sourde comme le miroir

de lait où chantent encore les cloches souveraines. (53)

découvrira que le chemin est tracé d'avance :

"Le jeu est sans rémission, dit l'enfant.

Déja avant de quitter, j'étais rendu.

Et nulle fièvre n'a pu convaincre d'impatience

le temps renouvelé." (54)

Maintenant le poète est arrivé à destination; il contemple l'absolu dans une sérénité conquise :

Calmes, calmes

au beffroi stellaire

calmes et tranquilies

tintent fleurs et glas

dans l'aveu insonore

des cloches d'autres mondes. (55)

L'articulation de la dernière partie intitulée «Dunes" se fait dans la récurrence des deux premières. Le poète révise sa démarche en toute sérénité :

Le regard erre dans l'œil.

II en est la ruse et la tendresse. (60) 
Et il raconte que "parmi les faveurs lancinantes de l'espoir et l'abondante nuit des sérénités" (60) un songe est né et qu'il en est mort. Ce songe pourrait bien être celui de l'identification avec l'absolu. De quel prix faut-il payer ce passage du songe à son incarnation?

Quel abîme inscrit illisible

par invitation de l'ombre

son effigie? (60)

Conscient ( «l'œil s'immole au regard"), il accepte de payer le prix de ce regard nouveau :

«La mort, dit-il, est soleil friable dans le creuset d'un or esseulé, moisson d'abeilles sur un miel océan. Proie d'intangible... » (61)

Alors que le poète est «à la charnière de l'interrogation», qu'il contemple un instant le chemin parcouru ( «je repose, dit-il») un sursaut de souffrance lui signifie la sécheresse de sa situation :

"L'amour s'est couché plus tôt que la mémoire dans les épines de l'abandon. » (62)

II constate :

Insonore et vacante

l'âme est le parcours

et la trame des saveurs. (62)

En rejoignant cette part de lui-même qu'il désire assumer jusqu'au bout,

"Dans l'oreille évasive des conques, j'écoute le temps inamovible, dit-il, dans l'arrimage de moi-même, dans la mesure des pas qu'un souffle annule.» (63)

II sait maintenant qu'il devra compter sur très peu de moyens pour continuer la route:

Ancré dans l'aire de son plaisir

le phénix, fleur des sables,

réjouit la solitude. (65)

II ose nommer ce nœud qui le préoccupe; et à partir de ce volet,

"Parmi les centres, dit-il, la vanité et l'audace du centre. Désavoué, le dernier mot est criblé de chaleur. » (66)

le poète habitera entièrement le geste créateur de son écriture («tel l'immobile dénoué en don d'immobilité »). Le poème devient «feuillet d'incendie» (67)

II accepte cette autre réalité :

"Mieux je sais, plus je mens, dit-il. Car le réel est un signe aboli, la trace du signe, la trace dans sa disparition. L'oubli dans le souvenir." (68)

II sait à partir de cette expérience vécue que «la vie s'écrit dans l'image sanguine d'une offrande" et que, s'il a pu faire le chemin à l'envers, seul «le poème disparaît » (p. 69) «d'un risque de mémoire» (p. 70). 
Après cette participation au cheminement spirituel du poète, une seconde lecture nous livre les clefs d'un monde encore peu exploré et qui s'avère d'un très grand intérêt. Une seconde approche du texte de Guy Lafond est donc possible, que j'intitulerai :

\section{Les Cloches d'autres mondes et la Résurgence de la mémoire : lectures croisées}

Les grands écrits ne sont-ils pas la résurgence des mythes anciens qui frappent à la porte de notre seconde mémoire? Ainsi en serait-il du livre-poème de Guy Lafond qui reprend en toute innocence les données fondamentales d'une mythologie indienne. Qu'on le veuille ou non, nous sommes habités par le sol qui nous a vus naître, qui a vu s'épanouir une culture façonnée par nos ancêtres. Ici, il s'agit bien de racines inconscientes qui jaillissent telles une lumière blanche, signifiant la source pure de l'inspiration : "La mémoire est un mal tenace... l'escale bruissante d'une parole aveuglée.» (p. 69) “L'amour s'est couché plus tôt que la mémoire dans les épines de l'abandon.» (p. 62). “Comme la mémoire escalade la mémoire. «Et mon œil bat au cillement heureux d'un risque de mémoire.» (p. 67). Je tenterai d'établir un parallèle en reprenant une à une certaines formules qui reviennent continuellement dans le corps du poème comme pour en étayer le sens mythique. Pour faciliter le cheminement aux lecteurs désireux de me suivre sur cette voie, j'utiliserai une seule source de référence: «Pieds nus sur la terre sacrée", textes réunis par T.C. McLuhan (Denoël-Gonthier, “Médiation»).

Telle la pensée de l'Indien, les Cloches d'autres mondes est habité par l'esprit du ciel et de la terre conjugués. Comme le dit si justement Mircéa Eliade : “Le sacré est camouflé dans une histoire et il opère. " Et l'Indien: "Quand j'ai eu dix ans, j'ai regardé la terre et les rivières, le ciel au-dessus et les animaux qui m'entouraient, et je n'ai pu m'empêcher de ressentir qu'ils étaient l'œuvre d'un pouvoir supérieur. J'étais si soucieux de comprendre ce pouvoir que j'interrogeai les arbres et les buissons ${ }^{2}$.»

Le poète prouve cette immanence et la traduit de la façon suivante:

L'œil est une étoile odorante

un sourire

un essaim d'adieux où rêve une présence

La nuit comble mon regard

et tout est loisir

l'arbre, la fête, l'âme. (21)

Dans la vie de l'Indien, il n'y a qu'un devoir inévitable - le devoir de prière - la reconnaissance quotidienne de l'invisible et de l'éternel. Ses dévotions quotidiennes lui sont plus nécessaires que sa nourriture de chaque jour [...] Après le bain, il reste dressé devant l'aube qui avance, face au soleil qui danse sur l'horizon, et offre sa prière muette [...] Le soleil du matin, la douce terre nouvelle et le grand silence, chaque âme doit les rencontrer seule! [...] Chaque fois 
qu'au cours de sa chasse quotidienne, l'homme rouge arrive devant une scène sublime ou éclatante de beauté - un nuage noir chargé de tonnerre avec l'arche étincelante d'un arc-en-ciel au-dessus d'une montagne, une cascade blanche au cœur d'une gorge verte, une vaste prairie teintée du rouge sang d'un couchant - il s'arrête un instant dans la position d'adoration ${ }^{3}$.

Guy Lafond commence son livre-poème par une offrande :

Équinoxe à l'orée des pistes.

L'aile est césure du ciel.

La mort est sans paliers. Je dérive parmi la nudité.

Et l'odeur musquée des redites.

Un champ s'endort dans la fleur.

Un spectre s'éveille dans le vent.

Tout est aérien, le vertige, les dés.

Le nœud mis en échec.

Le baiser. Un nuage, parfois, dans la main. (11)

Ce dialogue des éléments de la nature, source de prière pour l'Indien, est ici l'objet d'une contemplation intérieure. «Le chant, dit le poète, est un modèle d'espace à circonscrire" (p. 12) alors que pour l'Indien

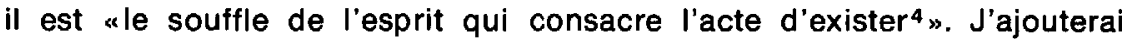
là-dessus, tirée des notes de Frances Densmore, la remarque suivante: "Dans toute étude de la pensée indienne, il faut prendre en considération ce qui n'aura pas été dit. L'expression entière et complète n'est pas coutumière chez l'Indien. L'élément non prononcé peut être l'objet d'une compréhension mutuelle dont rien ne transparaît dans les mots, ou peut être indiqué de manière telle qu'il n'est intelligible que pour ceux auxquels il s'adresse. II en est ainsi du “langage sacré» des “hommes-médecine» dans lequel les mots familiers prennent un sens occulte ${ }^{5}$."

Quatre références reviennent sans cesse dans le texte de Guy Lafond. Seraient-ce des messages anciens d'une mémoire collective inscrite dans le corps même du souvenir? Une hypermnésie, en quelque sorte? La question est posée. II est intéressant de noter que ces formules essentielles sont "objet de cultes" ou «symbole magique» chez l'indien : la pierre, le soleil, la mort et le cercle ou le circulaire auquel il faut joindre le centre. Cela est d'une évidence incontestable dans les préoccupations du poète telles qu'il les expose dans l'entretien.

Vous avez remarqué que toute chose faite par un Indien est dans un cercle, il en est ainsi parce que le pouvoir de l'univers agit selon des cercles et que toute chose tend à être ronde. Dans l'ancien temps lorsque nous étions un peuple fort et heureux, tout notre pouvoir nous venait du cercle sacré de la nation, et tant qu'il ne fut pas brisé, notre peuple a prospéré. L'arbre florissant était le centre vivant du cercle et le cercle des quatre quartiers le nourrissait. L'est donnait la paix et la lumière, le sud donnait la chaleur, l'ouest donnait la pluie et le nord, par ses vents froids et puissants, donnait force et endurance. Cette connaissance nous vint de l'outre-monde avec notre religion. Tout ce que fait le pouvoir de l'univers se fait dans un cercle. 
Le ciel est rond et j'ai entendu dire que la terre est ronde [...]. Le soleil s'élève et redescend dans un cercle [...]. Même les saisons forment un grand cercle dans leurs changements [...]. La vie de l'homme est dans un cercle de l'enfance jusqu'à l'enfance et ainsi en est-il pour chaque chose où le pouvoir se meut ${ }^{6}$.

Dans l'entretien qui précède, G. Lafond dit justement : «Mon expression deviendra circulaire à partir de ma troisième publication, l'Eau ronde." II faut aussi se rappeler les vers des Cloches d'autres mondes: «...le cercle, disponible aux fuites; la sphère aux miroirs» (p. 16); "Parmi les centres, dit-il, la vanité et l'audace du centre.» (p. 66).

Guy Lafond m'avouait dans une conversation qu'il réalisait ses ambitions poétiques à travers une perception circulaire du temps; mais c'est avec le jeu des éléments - pierre, soleil, mort - que le rapport de la pensée traditionnelle indienne et le vécu poétique de Guy Lafond est le plus efficace. Relevons quelques vers èt donnons ensuite la parole à l'Indien.

La rose est une pierre comblée de son excès. (17)

Faste de la pierre qui ne termine jamais

Ronde et lisse comme le bonheur. (43)

Le vent s'abrège en plumes de pierre l'immense peuple de noms l'intraduisible (62)

La mémoire assiégée est la blessure des pierres (63) «... dans l'écume le courroux acide des pierres. » (64)

La pierre dit que par ma quête je m'étais montré digne d'une aide surnaturelle [...] Comme le soleil et la lune, ces pierres sont rondes et nous savons que toutes choses rondes ont un lien entre elles [...]. Toute ma vie je suis resté fidèle aux pierres sacrées. J'ai vécu en accord avec leurs exigences et elles m'ont assisté dans toutes mes peines. J'ai tenté de me rendre capable, tout seul, de manier ces pierres sacrées aussi bien que possible; mais je sais que je ne suis pas digne de parler à l'esprit. Je fais mes demandes aux pierres et elles intercèdent pour moi auprès de lui ${ }^{8}[\ldots]$. Quand un homme-médecine dit qu'il parle aux pierres sacrées, c'est parce que de toutes les substances du sol, elles sont celles qui apparaissent le plus souvent dans les rêves et qui peuvent communiquer avec les hommes ${ }^{9}$.

On sait que les pierres sont considérées comme objet de culte chez plusieurs Indiens. Guy Lafond voit, ou plutôt ressent la mémoire assiégée comme une blessure faite aux pierres. Par analogie, son texte serait le dévoilement du sens sacré de sa perception poétique.

Le soleil tient aussi une place particulière dans l'œuvre récemment publiée par le poète. Rapprochons à nouveau sa parole de celle de l'Indien.

Soleil d'outre azur. Le ciel partout à la rencontre d'un ciel. (17)

L'arrogance du regret a couché le soleil (22)

... le soleil résonne comme un soupir (25)

... un soleil noir est le vaisseau du jour (26)

L'épée du soleil s'accorde aux neiges blondes (64) 
Le soleil est plaie d'incandescence (67)

"L'arbre s'évanouit dans le signe, comme le soleil dans l'agonie du feu... (67)

Un vieux Sioux Téton explique que son peuple croyait en un pouvoir mystérieux dont la plus grande manifestation était la nature, le soleil étant une de ces représentations [...]. Nous avons sacrifié au soleil et nos souhaits ont été exaucés ${ }^{10}$.

Toute créature vivante, tire sa vie du soleil. Si le soleil n'était pas, ce serait la nuit et rien ne pousserait - la terre serait sans vie ${ }^{11}$.

La danse du soleil est si sacrée pour nous que nous n'en parlons guère... La lacération des corps pour acquitter les vœux de la danse du soleil est différente de la lacération de la chair chez les gens dans le deuil [...]. Je puis donner du tabac ou d'autres objets au cours d'une danse du soleil, mais si je garde le meilleur, qui pourra croire que je suis sincère? Nous devons tous reconnaître l'œuvre d'un plus puissant que l'homme. Le plus grand de tous est le soleil sans lequel nous ne pourrions vivre ${ }^{12}$.

Clifford Geertz remarque que pour la majorité des Oglalas, [...] la danse du soleil, le cercle est un symbole lumineux [...] dont le sens est perçu intuitivement et non consciemment interprété... Très souvent, l'idée d'un cercle sacré, comme forme naturelle ayant une portée morale, engendre des sens nouveaux [...]; elle relie des éléments de leur expérience qui sembleraient tout à fait disparates et incompréhensibles sans elle ${ }^{13}$.

\section{Or Guy Lafond écrit aussi :}

L'étoile du matin est l'axe de soleils reconciliés

l'intime allégorie des morts. (36)

Il me paraît donc évident que l'élément primordial de l'inspiration du poète est en relation étroite avec les différents concepts de la nature tels que vécus par l'Indien. Je mentionnerai enfin la construction du livre qui se divise en quatre parties, prologue compris, ce dernier se déroulant à son tour en quatre temps:

«Je hisse mon nom sur l'axe du chiffre» (p. 14) «Or, rapporte McLuhan, pour la grande majorité des tribus d'Amérique du Nord, le nombre quatre est un nombre sacré. [...] Ceci parce qu'ils reconnaissent quatre directions [...], quatre divisions du temps [...], quatre parties dans toutes choses qui poussent sur le sol [...], quatre sortes de choses qui respirent [...], quatre choses au-dessus du monde [...], quatre periodes dans la vie d'un homme [...]. Puisque le Grand Esprit a fait aller toute chose par quatre, l'homme devrait faire le plus de choses possibles par quatre ${ }^{14}$.

Je terminerai ici ce court aperçu des affects de la mémoire en signalant la subtile emprise qu'exerce la mort dans le monde poétique de Guy Lafond.

Le poète ne s'embarrasse pas du choix superflu. Dès sa première publication, il signifie ce que sera ce choix: «J'ai choisi la mort." Mais, comme pour l'Indien : “Morts, ai-je dit? Mais il n'y a pas de mort! Seulement un changement de monde ${ }^{15}$." 
Si le poète écrit, c'est bien pour traduire le changement qui s'opère dans sa conscience : "La vie n'existe pas, elle s'irradie." (p. 13).

\section{Peut-on apprivoiser la mort?}

\section{Belles dents de la mort}

Violence inespérée du néant

Et sous la paupière du jour

L'écume éclatante de la nuit reconnue (45)

Par comparaison, I'Indien cultive un rapport tout aussi particulier avec la mort. Preuve en est donnée par ce texte qui garde toute l'épaisseur du mystère indien :

Je sais que notre peuple possédait des pouvoirs remarquables de concentration et d'abstraction, et je me demande parfois si le fait d'être aussi proche de la nature, [...] garde l'esprit sensible aux impressions peu communément ressenties, et en contact avec les pouvoirs invisibles. Certains d'entre nous semblaient avoir une singulière intuition quant à l'emplacement d'une tombe; ils l'expliquaient en disant qu'ils étaient entrés en communication avec l'esprit du défunt. Ma grand-mère était de ce nombre, et aussi loin que je me souvienne, lorsque nous campions dans un pays inconnu, mon frère et moi cherchions et trouvions des ossements humains à l'endroit qu'elle nous avait indiqué comme étant un lieu de sépulture ou l'emplacement où un guerrier isolé était tombé. Bien sûr, tout signe extérieur de sépulture était depuis longtemps effacé ${ }^{16}$.

Pour Guy Lafond, "La mort est lieu de mort" (p. 67) ou «La mort allège l'amour / la nuit trop ancienne des aurores" (p. 27).

L'Indien explique ainsi son attachement à la terre: "Les cendres de nos ancêtres sont sacrées et l'emplacement où ils reposent une terre sanctifiée 17 ." "Nos morts n'oublient jamais le monde merveilleux qui leur a donné la vie ${ }^{18}$." Guy Lafond, lui, compare la mort au soleil : "La mort, dit-il, est soleil friable." (p. 61).

Maintenant, nous pouvons nous poser l'ultime question: qu'est-ce que la mort? Peut-être trouverions-nous une certaine réponse à cette grave interrogation en essayant de changer notre approche de la vie? De toute évidence l'Indien possédait le secret et l'art de vivre qui rendaient la mort acceptable: «Les désirs d'un homme doivent tendre vers l'authentique, non vers l'artificiel ${ }^{19}$. "Ce à quoi le poète répond: "La mort est sans palier» (p. 11), «L'excédent est mortel» (p. 13), «il faut mourir à l'unanime» (p. 16), "la mort est le sceau de son image» (p. 65). 
1. Tous les chiffres entre parenthèses renvoient aux pages du recueil commenté, les Cloches d'autres mondes, Montréal, Hurtubise-HMH, "Sur parole», 1977. [Tous les soulignés sont de Guy Lafond.]

2. Pieds nus sur la terre sacrée, p. 27.

3. Ibid., p. 49.

4. Ibid., p. 36.

5. Ibid., p. 197.

6. Ibid., p. 55-56.

7. Voir l'entretien qui précède.

8. Pieds nus sur la terre sacrée, p. 28.

9. Ibid., p. 30.

10. Ibid., p. 29.

11. Ibid.

12. Ibid., p. 52.

13. Ibid., p. 200 .

14. Ibid., p. 42.

15. Ibid.

16. Ibid., p. 19.

17. Ibid:, p. 37 .

18. Ibid., p. 41.

19. Ibid., p. 31.

20. N.D.L.R. : L'auteur de ce commentaire et de l'entretien qui précède a récemment publié Une mémoire déchirée, récit autobiographique, chez Hurtubise$H M H$, "L'arbre". Elle a également fait paraître, en 1946, les Sables du rêve, aux Cahiers de la file indienne, dont nous avons déjà parlé (voir Voix et images, vol. II, $n^{\circ} 1$, septembre 1976, "le Rire de la reine " par P. Haeck et C. Savary). 